\title{
Application of active quenching of second generation wire for current
}

\section{limiting}

Vyacheslav F. Solovyov ${ }^{1}$, andQiang $\mathrm{Li}^{1}$

${ }^{1}$ Condensed Matter Physics and Materials Science Department, Brookhaven National Laboratory, Upton, NY11973

\begin{abstract}
Superconducting fault current limiters (SFCL's) are increasingly implemented in the power grid as a protection of substation equipment from fault currents. Resistive SFCL's are compact and light, however they are passively triggered and thus may not be sufficiently sensitive to respond to faults in the distribution grid. Here, we explore theprospect of adding an active management feature to a traditional resistive SFCL.A flexible radio-frequency coil, which is an integral part of the switching structure, acts as a triggering device. We show that the application of a short, $10 \mathrm{~ms}$, burst of acmagnetic field during the fault triggers a uniform quench of the wire and significantly reduces the reaction time of the wire at low currents. The ac field burst generates a high density of normal zones, which merge into a continuous resistive region at a rate much fasterthan that of sparse normal zones created by the transport current alone.
\end{abstract}




\section{Introduction}

Today's electrical grid is transforming in a way that can be described as separation of generation from transmission[1].A recent survey of global electricity providers indicates that up to $75 \%$ of utilities consider incorporation of independent distributed generation as their top challenge[2]. It is expected that in the coming yearslarge numbers of distributed generating units will be installed on sites where previously little generation existed.

Integration of multiple distributed power sourcesinto the existing power grid can increase the frequency and magnitude of short-circuit eventsboth at high-voltage and medium/low-voltage levels. This explains the recent interest in fault current limiting technologies[3].If a fault current limiter (FCL) can provide the required protection, a utility can forgo the substation upgrade needed to handle higher fault currents and avoid a substantial capital expense $[4,5]$.

Superconducting FCL's (SFCL) attracted interest after the advent of hightemperature superconducting materials enabled economic operation of SCFLS's at liquid nitrogen temperatures [6]. The availability of second generation $(2 \mathrm{G})$ wire with the critical current, $I_{c}$, of $>200 \mathrm{~A} / \mathrm{cm}$-width at $77 \mathrm{~K}$ spurred further progress of the SFCL technology [7-10]. Recentlya $2 \mathrm{G}$ wire-resistive SFCL with record power ratings $(138 \mathrm{kV}$ @ $0.9 \mathrm{kA}$ ), was designed, built[11] and tested [12]by the Siemens-Nexans-AMSC team. Operation of a resistive SFCL can be described as a current-induced unprotected quench. The superconducting taperapidly becomes normal (resistive) when the current $I_{c}$. There are several limitations of resistive SFCL technology. The $I_{c}$ of mostapplied superconductors is not uniform, consequently not the whole length of the conductor 
becomes normal as the current becomes higher than $I_{c}$. There exists the risk of local overheating and irreversible damage to the wire due to the partial transition of the wire in a resistive SFCL. Additionally, in many distribution substation applications, it is desirable to actively pre-set the FCL trigger to a level as low as $125 \%$ of the nominal current. Such a precision is difficult to achieve with a traditional resistive FCL, because the safe nominal operating peak current is typically set below $80 \%$ of $I_{c}$.In contrast a semiconducting FCL can be actively managed by turning off semiconducting switches (such as gateturn-offthyristors or insulated bi-polar transistors)[13]. This allows for precise setting of the fault current trigger at any value. The active management feature of an FCL is especially attractive for grids with high content of distributed generation, because magnitude of the fault depends on the number of generators connected to the grid at a given moment [14].

Here we utilize the concept of RF-assisted switching [15]in order to demonstrate active current limiting and uniform quenching of a commercial $2 \mathrm{G}$ wire at a current level of $120 \%$ of $I_{c}$. First, we evaluate the response of the $8602-$ FCL stainless-steel stabilized 2G wire (AMSC Corp.) to the over-current, i.e. current level higher than the $I_{c}$ of the wire. Then, we measure the superconducting-normal state transition kinetics under application of high-frequency magnetic field. Finally, we demonstrate the advantage of RF-assisted current limitation in a small-scale FCL. 


\section{Experiment}

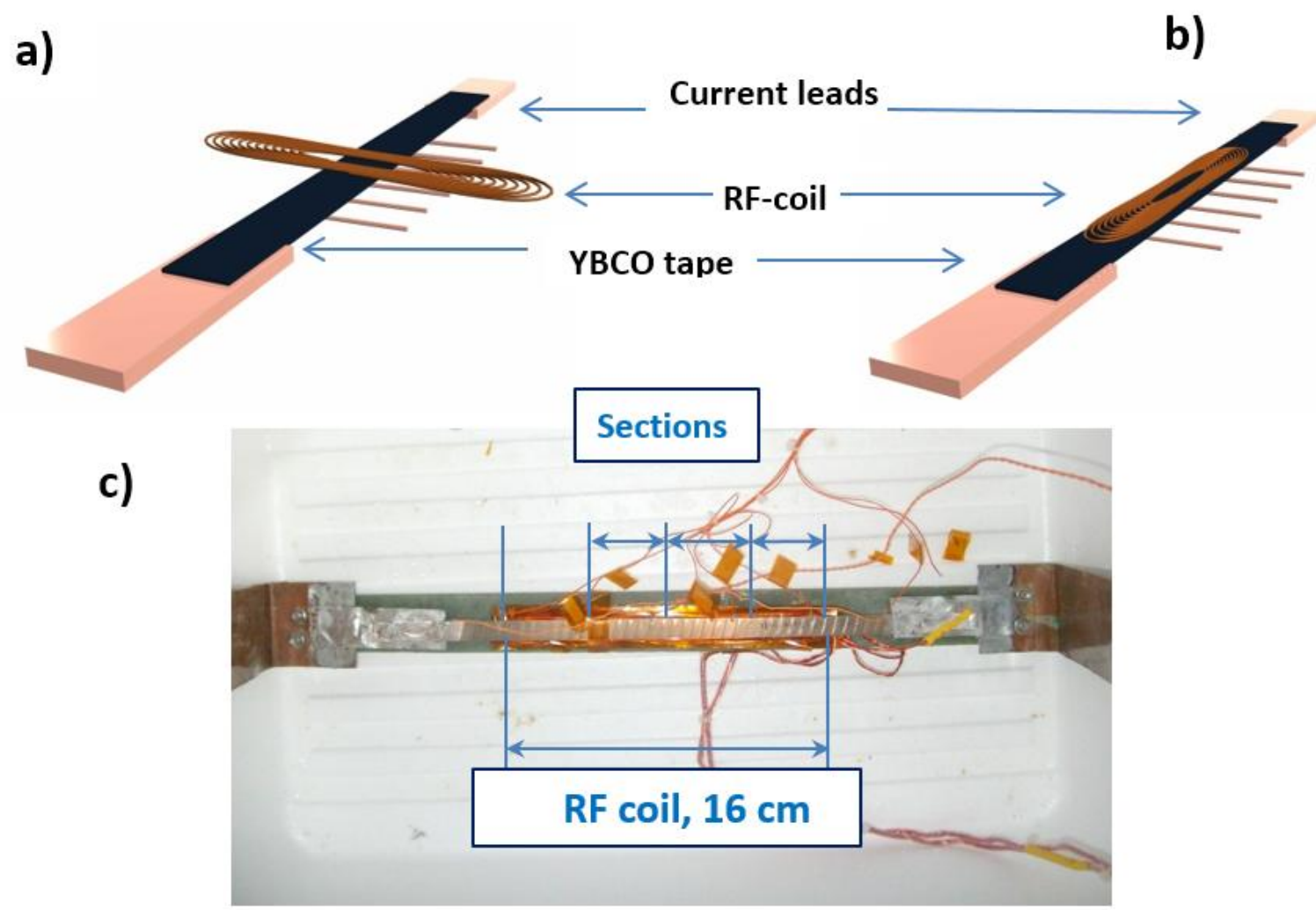

Fig.1. Conceptual rendering of the experiment in a) Normal zone propagation measurement configuration; b) Full-length excitation configuration. c) A photograph of the full-length excitation setup, showing the $2 \mathrm{G}$ wire tape coupon, voltage leads and the RF coil.

\subsection{Short sample quench dynamics}

We used the standard high-resistance wire (8602-FCL) offered by the AMSC Corp. The wire is a $10 \mathrm{~mm}$ wide YBCO-RABiTS tape soldered between two $12 \mathrm{~mm}$ wide layers of $75 \mu \mathrm{m}$ thick $316 \mathrm{~L}$ stainless steel foil. The average critical current density of the wire, measured by the DCtransport method in $520 \mathrm{~cm}$ coupons, was $260 \pm 20 \mathrm{~A}$. 
Fig.1schematically shows the experimental setup used in evaluating the response of a short, $20 \mathrm{~cm}$ coupon of the tape.The tape was soldered to copper current leads and the whole assembly was placed in a Styrofoam bath filled with liquid nitrogen.Voltage leads were soldered to the tape 1-4 cm apart, thus allowing for recording voltage in four long sections numbered 1 through 4, see Fig.1c. A digital data acquisition system (LabView DAQ) recorded the differential voltage during the overcurrent application. The same system synchronized the application of the power to the flat RF coil and the transport current.The transport current was delivered by a Sorensen 10 V/ 1,200 A power supply.

The RF field was generated by a flatcoil that was comprised of 8 turns of gauge 14 Liz wire wound to conform $16 \mathrm{~cm}$ long and $1 \mathrm{~cm}$ wide race-track shape. The coil was placed directly under the tape in two configurations: (i) Fig.1a, the normal zone (NZ) propagation velocity (NZPV) configuration, where the coil-inducedquench was restricted to the middle of section \#3 of the wire.; (ii) Fig.1b, full-length excitation configuration, where the coil-induced quench covered in the entire $20 \mathrm{~cm}$ length of the conductor.We determined experimentally that $100 \mathrm{~ms}$ of $100 \mathrm{~W}$ RF signal is sufficient to create a uniform NZ in section \#3. In the NZPV experiment, a transport current below the $I_{c}$ value, $260 \mathrm{~A}$, was applied to the tape. A $100 \mathrm{~ms}$ long burst of RF power was supplied directly to the RF coil creating a NZ in section \#3, see Fig.1c. The NZ propagation to the neighboring sections, \#1 and \#2 (1.5 cm apart), was detected by development of voltage on the corresponding voltage taps after the RF signal was turned off. We note that this method does not involve heating $[16,17]$ of the superconductor, thus eliminating the effect of the external heat load. 
In the full-length excitation configuration, shown in Fig.1b, the coil excited the full length of the tape. We explored two regimes: (i) Passive regime, where current exceeding 260 A was applied to the tape and the voltage in sections \#1-4 (4 cm apart) was recoded as a function of time; (ii) RF-assisted or active regime, where application of the current was also accompanied by a $10 \mathrm{~ms}$ long, $500 \mathrm{~W}$ RF signal applied to the RF coil. The section voltage was recorded as a function of time. The power supply voltage was set at 2 $\mathrm{V}$, which correspondsto $0.125 \mathrm{~V} / \mathrm{cm}$ electric field strength in the current-limiting regime. A limited number of tests were performed at the electric field strength level of $0.5 \mathrm{~V} / \mathrm{cm}$.

\subsection{Active FCL prototype}

A small-scale FCL prototype was assembled from $6 \mathrm{~m}$ of $2 \mathrm{G}$ wire wound in a bifilar manner on a $15 \mathrm{~cm}$ coil. A flexible RF coil, comprised of two strands of the Litz cable was sandwiched between the superconducting tapes, see Fig.2a. The coil used in the FCL prototype can be described as one extended loop of multi-strand cable, Fig.2b. The design is different from the race-track configuration used in the small-scale switching experiments. The change in design was necessary in order keep the resonant frequency of the RF coil circuit in the $100-200 \mathrm{kHz}$ range. The resulting normal state resistance of the FCL assembly was shown to be $0.6 \Omega$. (Fig.2cshows the assembled FCL with attached current and voltage leads). The coil was suspended from an insulating top flange. The top flange provided the mounting surface for the impedance matching components, current lead and the RF power feed.

The RF coil was driven by an H-bridge inverter, the schematics of which are shown in Fig.3a. A $2 \mathrm{mF}$ capacitor (Vishay) was connected in series with the RF coil, L1, forming a $\approx 200 \mathrm{kHz}$ resonant circuit. An inductance, L2, matched the impedance output 
of the inverter with that of the L1-C1 circuit. The inductance L2 was comprised of 2 turns of the Litz cable over $5 \times 2 \mathrm{~cm}$ ferrite core. The number of turns was adjusted so that the power supply, H1, delivered the full power, $200 \mathrm{~W}$, into the resonant circuit L1-C1. The impedance-coupling elements, $\mathrm{L} 2$ and $\mathrm{C} 1$, were mounted directly on the flange for easy adjustment, Fig.3b. The whole assembly was designed to fit in an 8" ID cryostat filled with liquid nitrogen.

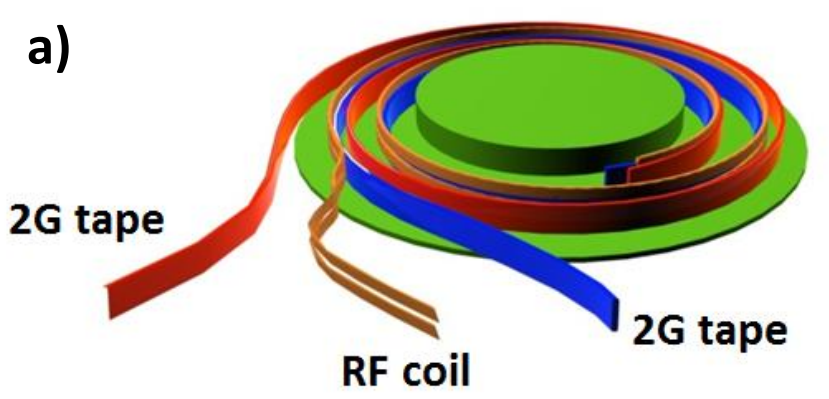

b)

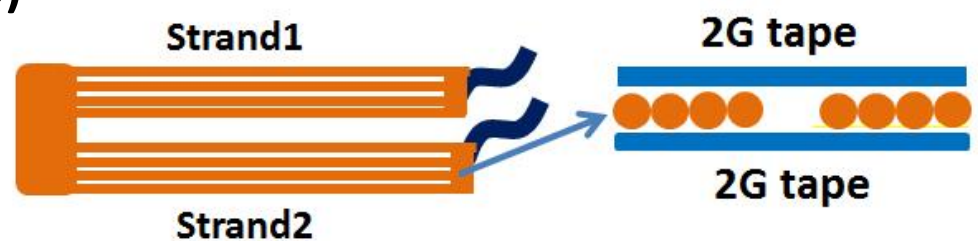

c)

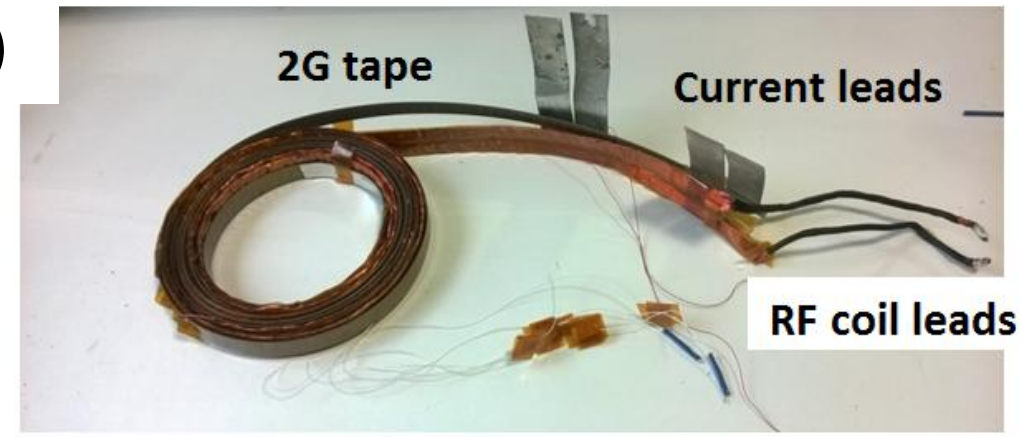

Fig.2. a) Schematic rendering of the RF-assisted FCL prototype, showing the RF coil sandwiched between two $2 \mathrm{G}$ tapes. The RF coil is comprised of four multi- 
strand Litz wires. b) Schematic drawing of the RF coil geometry. c) The FCL assembly, showing the tape, current leads and RF coil leads.

The prototype FCL was tested at $30 \mathrm{~V}$, up to $500 \mathrm{~A}$. The power supply was set in the current mode with the voltage limit at $30 \mathrm{~V}$. During the tests, we used the same sequence of DC current and RF power application as in the short-sample experiments: both the DC current and the RF signal were applied simultaneously and the time profile of voltage and current were recorded over a $10 \mathrm{~s}$ time interval.

a)
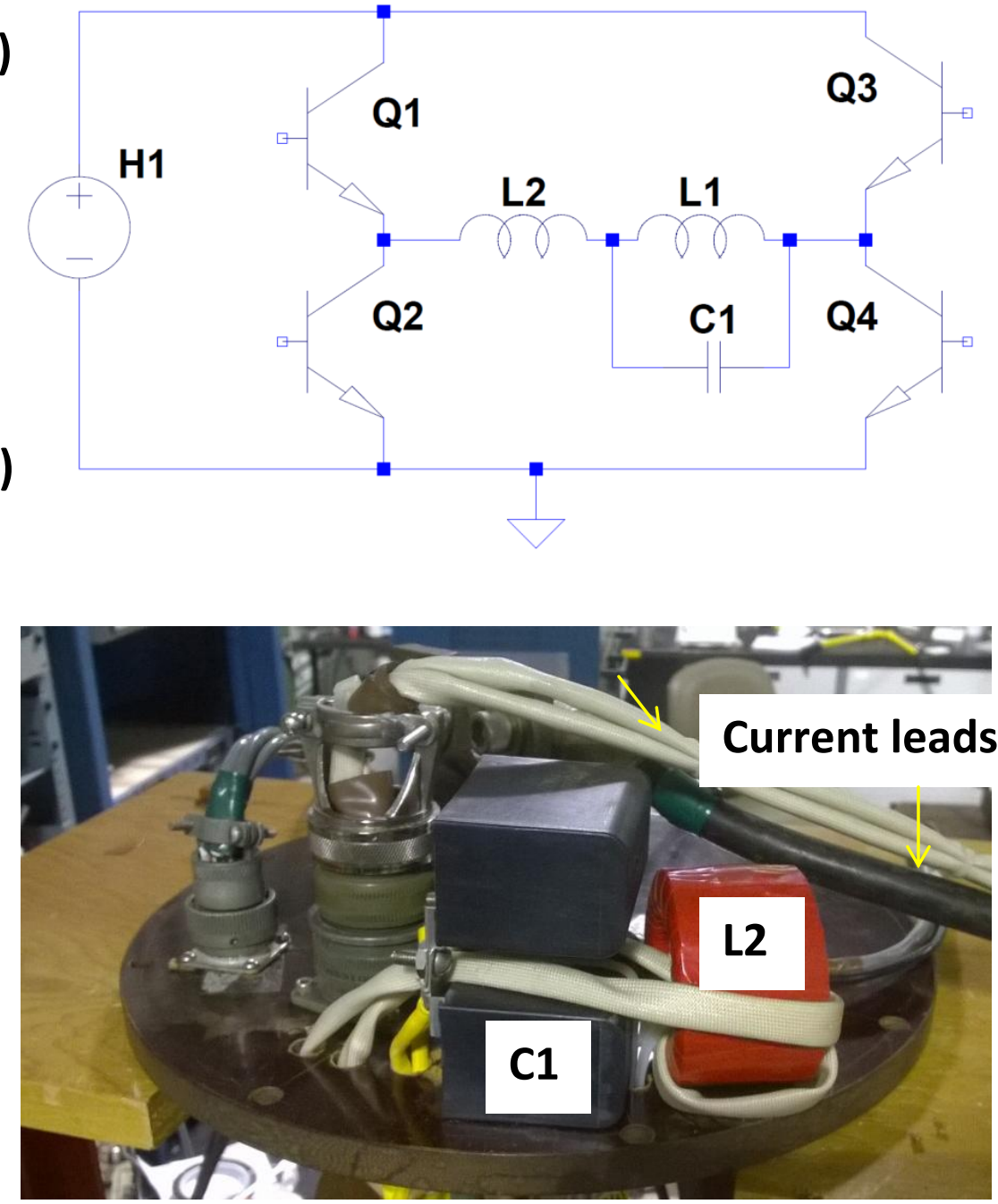
Fig.3. a) Schematic of the RF drive. b) Impedance-matching elements mounted on the top flange. Inductance L1 is the RF coil shown in Fig.2c.

\section{Results}

3.1 Passive quench of a short tape sample

a)

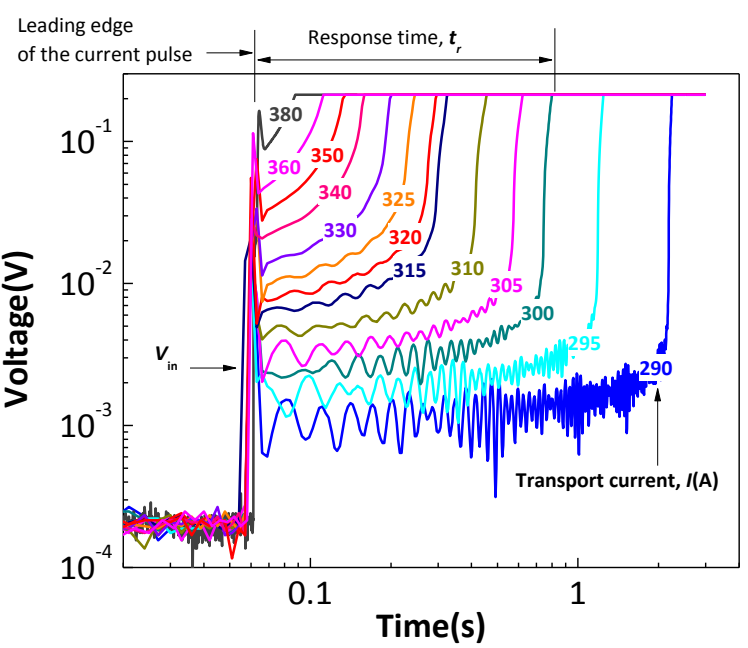

b)

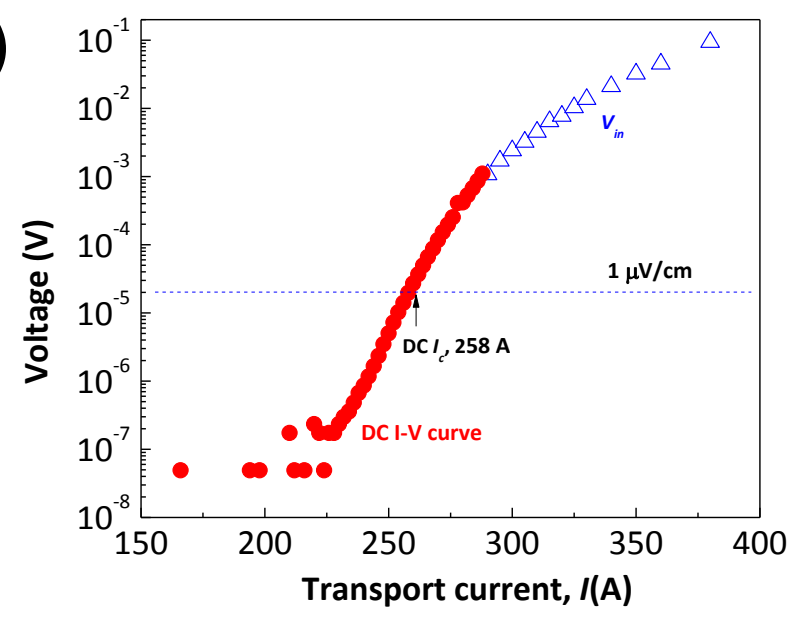

Fig.4. a) Response of the $20 \mathrm{~cm}$ wire coupon (see Fig.1) to the current pulse. The numbers on each curve indicate the current value; b) combination of the DC I-V curve (fullcircles) and the initial voltage, $V_{i n}$, across the tape after application of the current pulse (opentriangles). 
Fig.4a shows the response of the $2 \mathrm{G}$ wire to the step-wise overcurrent in the passive regime. Immediately after application of the current pulse we observe an initial voltage step, $V_{i n}$. The voltage across the tape continues to rise gradually until after a time period, $t_{r}$, then the tape resistance rapidly approaches the normal state level. At this point the voltage across the tape is high enough so that the current is limited by the tape resistance. We arbitrarily define $t_{r}$ as the response time, i.e. time required for the onset of the current-limiting regime and the full-length quench of the tape. Fig.4b superimposes the DC $I-V$ curve of the tape and $V_{i n}$ as a function of the applied current. One can easily infer from the plot that the $V_{i n}(I)$ continues the DC $I-V$ curve. Fig.4a shows that at $320 \mathrm{~A}$ $\left(120 \%\right.$ of $\left.I_{c}\right)$ it takes hundreds of milliseconds to attain current limiting.

\subsection{Normal zone propagation velocity measurements}

In order to better quantify the NZ dynamics, we measured the normalzone propagation velocity (NZPV) using the configuration shown in Fig.1a. Fig.5a shows time profiles of the voltage over sections \#1 and \#2, after application of the RF signal. The plots clearly demonstrate the dynamics of the NZ propagation through the tape with welldefined propagation time intervals. We calculate the average NZPV by dividing the average section length $(1.5 \mathrm{~cm})$ by the average propagation time. Fig.5b shows the dependence of the propagation velocity on the critical current density. The solid line is

the approximation $\sim I^{2.5}$, where $I$ is the DCtransport current through the tape. We clarify that the central section \#3 was excited by the RFsignal for $100 \mathrm{~ms}$, thus the NZ propagation through zones \#1 and \#2 was driven only by the transport current. 


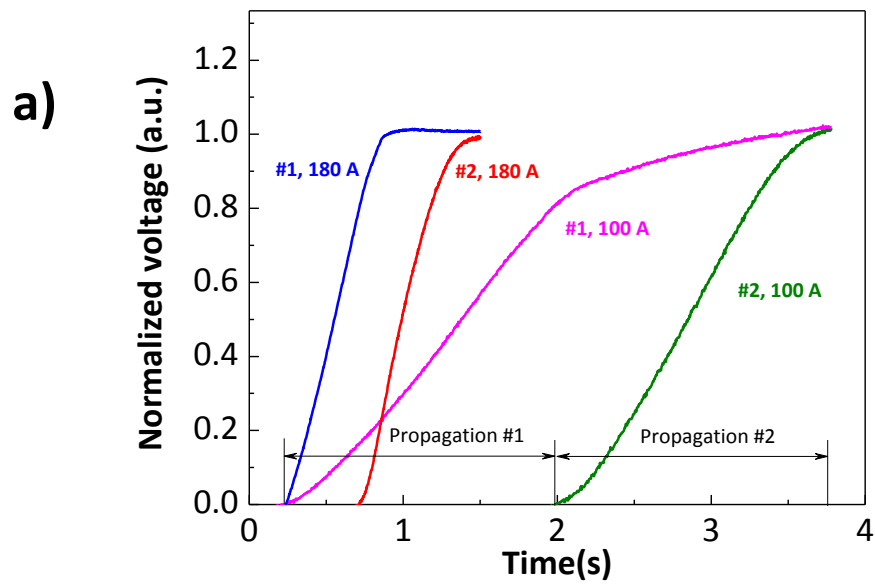

b)

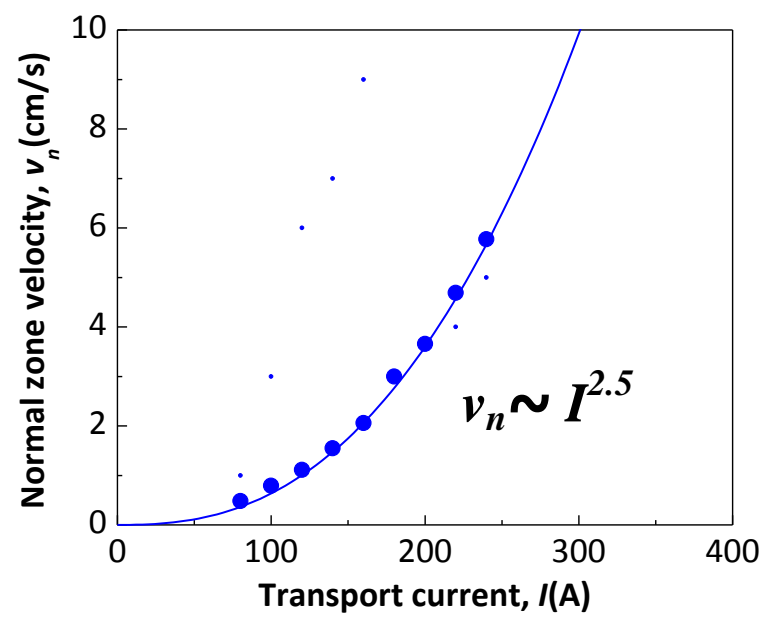

Fig.5. a) Normal zone propagation at $180 \mathrm{~A}$ and $100 \mathrm{~A}$ transport current. The initial normal zone was generated by application of 100 msRFsignalpulse in section \#3, seeFig.1a. The arrows show the propagation time for sections \#1 and \#2 (sections are $1.5 \mathrm{~cm}$ long); b) The normal zone propagation velocity as a function of transport current. The solid line is the power law fit, $v_{n} \sim I^{2.5}$.

\subsection{Uniformity of passive and RF-assisted quench of a short tape coupon}

During the full-length excitation experiments the RF coil is exciting the full length of the tape, Fig.1b,c. We limited the power supply voltage to $2 \mathrm{~V}$, which corresponds to the 
electrical field strength of $0.125 \mathrm{~V} / \mathrm{cm}$. In the normal state the wire resistance is measured to be $0.015 \Omega$, corresponding to current limiting at $\approx 115 \mathrm{~A}$ level if the entire wire coupon were normal.Fig.6compares the time profiles of the section voltage and the transport current in the passive overcurrent regime (Fig.6a) and assisted by 10 msRFpulse, Fig.6b. In the passive regime a noticeable voltage rise in sections 1 and 4 is observed after 200 msof the current application, while sections \#2 and \#3 are reacting at a much slower rate. Even after $1 \mathrm{~s}$ of overcurrent application there is still a large difference between the voltages of sections\#4 and \#3.

a)

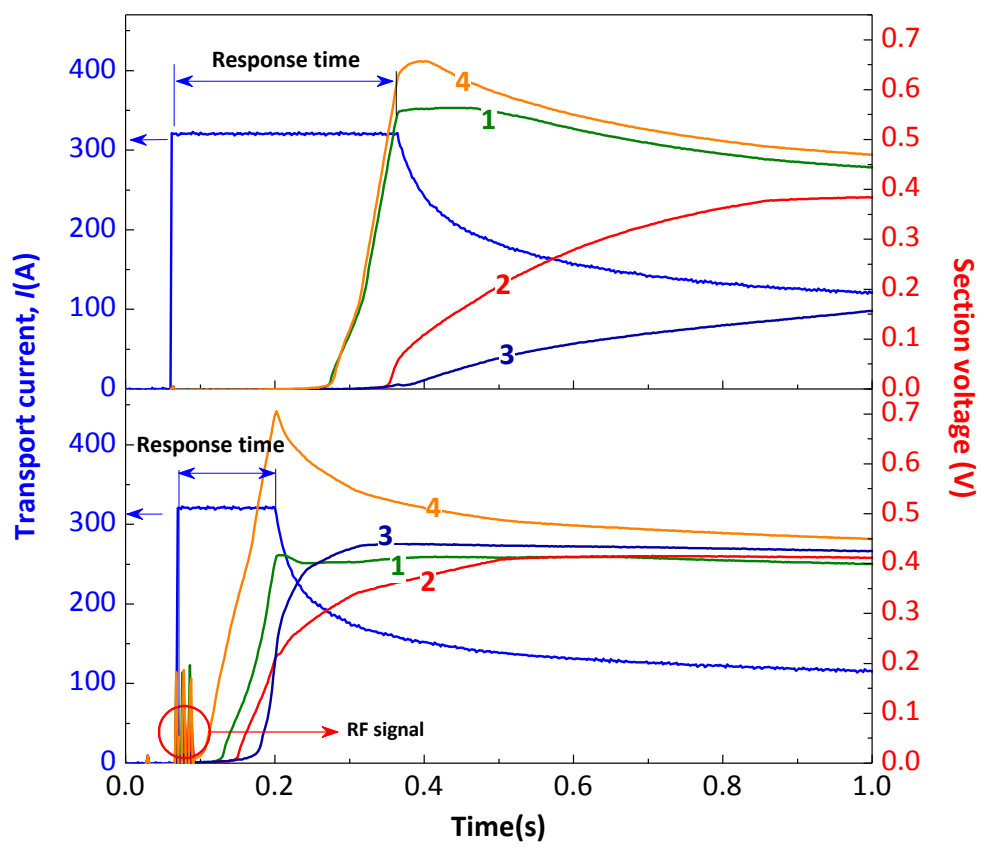

Fig.6. Time profiles of the section voltage of the $16 \mathrm{~cm} 2 \mathrm{G}$ wire coupon after application of 320 A current, see Fig.1c for the section layout. The numbers adjacent to the curves are the section numbers. a) Passive response of the wire; b) Response assisted by application of $10 \mathrm{msRF}$ signal. The RF signal waveform is superimposed on the tape voltage profile. 
Fig.6b illustrates the improvement of the response if the quench is assisted by the RF signal. We observe significantly better uniformity; all the four sections develop a normal zone within $10 \mathrm{~ms}$ of the RF pulse application. We also note that the voltage is equally distributed between the tape sections. The response time, defined as the onset of the current limiting is reduced by a factor of two.

Due to the uniformity of the RF-assisted quench the wire sustained an electric field strength up to $0.5 \mathrm{~V} / \mathrm{cm}$. The critical current density of the tape was measured before and after the RF-assistedquenching experiments at $0.5 \mathrm{~V} / \mathrm{cm}$. Fig.7a compares the $I-V$ curves of the as-received tape coupon (closed symbols) and the same tape samples after 20 cycles of quenching (open symbols) with electric field strength up to $0.5 \mathrm{~V} / \mathrm{cmapplied}$ for $5 \mathrm{~s}$. We observe no detectable change of the $I-V$ curve, which confirms that the RFassisted quenching does not damage the tape.In contrast, an attempt to perform a passive quench at $0.5 \mathrm{~V} / \mathrm{cm}, 320 \mathrm{~A}$, resulted in failure of sections \#4 and \#1. A snapshot of a video shot during the passive quench experiment,Fig.7b, clearly shows bright glow in sections 1 and 4 which develops after 500 msupon current application. Subsequent inspection revealed of the tape revealed that in the affected areas the stabilizer was completely delaminated from the superconductor. 

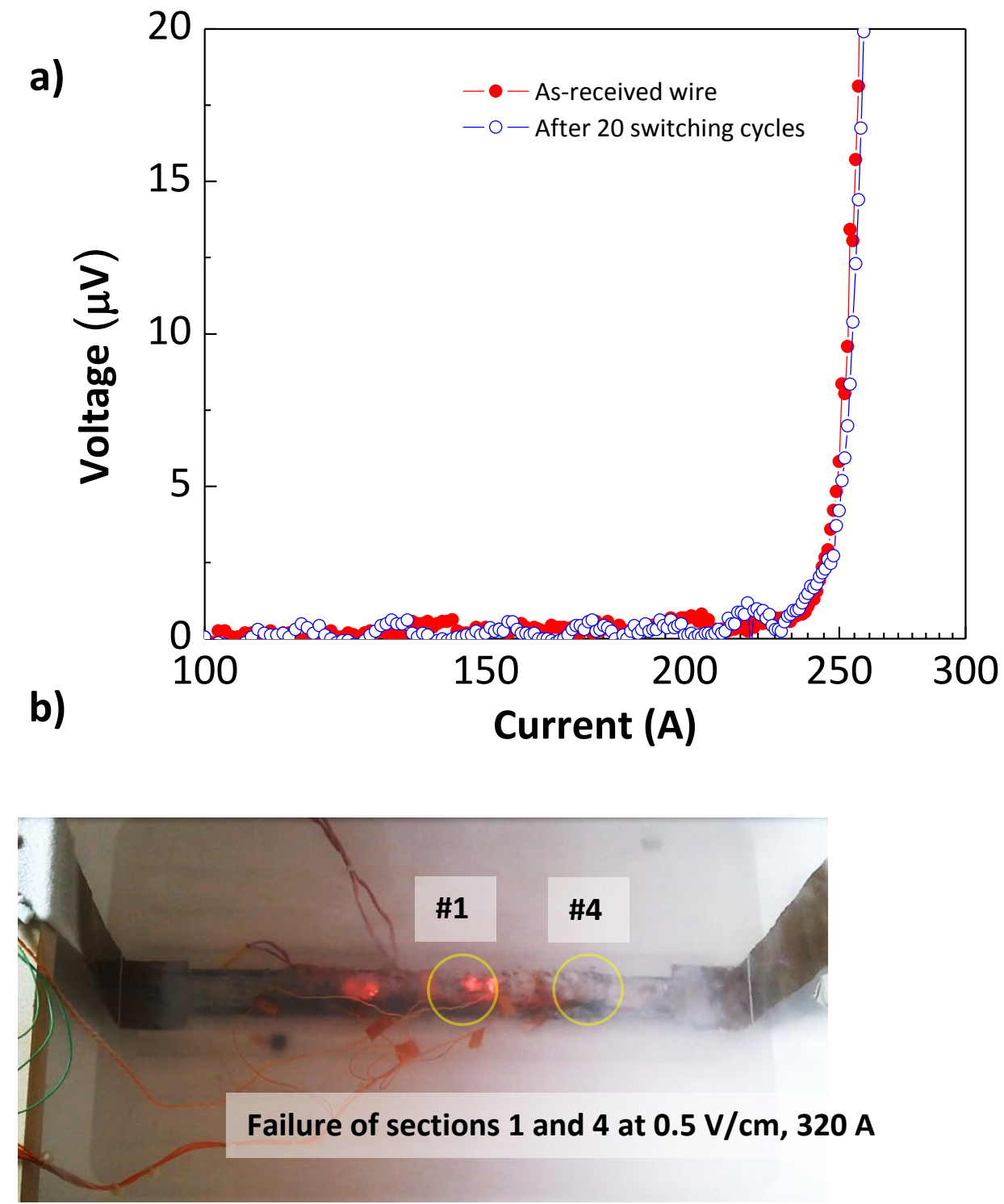

Fig.7. a) $I-V$ curves of the as-received tape are compared with the $I-V$ curve of the same tape after 20 cycles of switching 320 A current, up to $0.5 \mathrm{~V} / \mathrm{cm}$. We observe no $I_{c}$ degradation due to the tape RF-assisted quench. b) Photograph of the wire immediately after sections \#1 and \#4 failed during the passive mode quench at 0.5 $\mathrm{V} / \mathrm{cm}$. 
a)

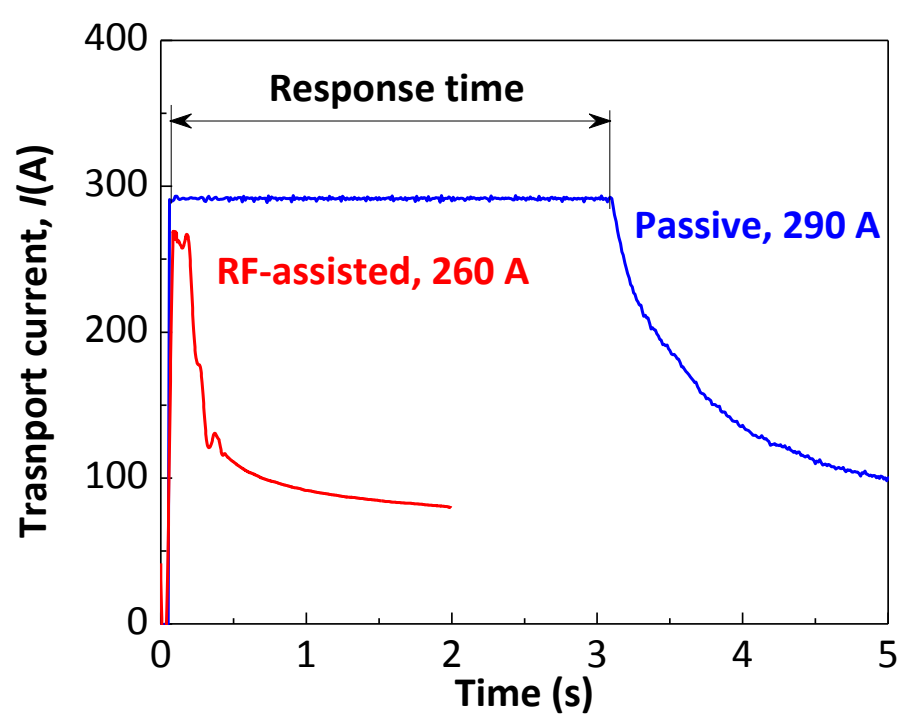

b)

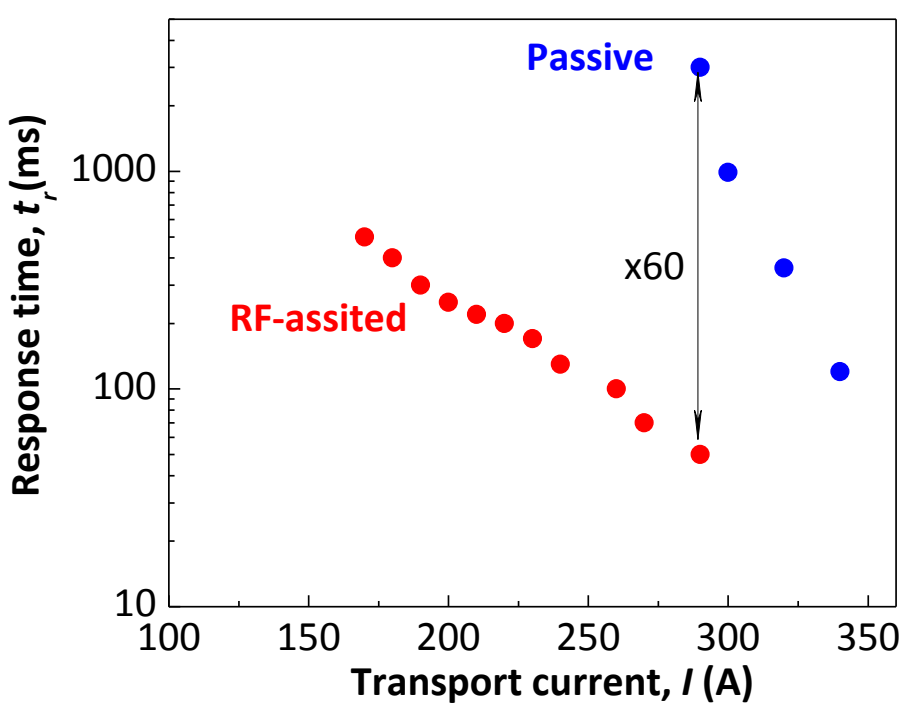

Fig.8. a) Comparison of the current limiting reaction of the FCL prototype in active and RF assisted regime. b) Response time of the FCL prototype in passive and RFassisted regime.

Fig. 8a compares time profiles of transport current through the FCL coil in passive and RF-assisted modes (10 ms, $200 \mathrm{~W}$ burst). The addition of the RF burst dramatically 
reduced the response time of the device: the current limiting starts after $<50 \mathrm{~ms}$ of the RF bust application.Fig. 8bsummarizes the FCL tests by presenting the current dependence of the response time. We define the response time as the on-set of current limiting, see Fig. 8a. As see from the figure, the RF assistance improved the response time by a factor of 60 .

\section{Discussion}

The highly-nonlinear $I-V$ characteristic of a superconductor can be utilized in currentlimiting and power conditioning applications. However, unlike semiconducting devices, where the $I$-Vcurve is determined by the predictable band-gap, the $I_{c}$ value of a superconductor depends on many intrinsic and extrinsic factors. Moreover, an applied conductor cannot be adequately described by a single $I_{c}$ value; rather one should use a statistical distribution of $I_{c}$ values along the length of the conductor[18, 19]. Traditionally $I_{c}$ is identified at the onset of dissipation at an electric field strength of $1 \mu \mathrm{V} / \mathrm{cm}$. The $I_{c}$ value in a non-uniform wire becomes less certain since it depends on the separation between the voltage taps[20]. Nevertheless, the voltage drop over a $100 \mathrm{~m}$ coupon at 1 $\mu \mathrm{V} / \mathrm{cm}$ is only $10 \mathrm{mV}$, assuming that the whole length of the wire is dissipating uniformly.The maximum reversible operating electric field strength for a coated conductor is $\approx 1 \mathrm{~V} / \mathrm{cm}[21]$. Therefore, we can define the onset of the current-limiting response of a superconducting wire at a dissipation level to be greater than $0.1 \mathrm{~V} / \mathrm{cm}$. This simple estimate shows that the FCL response at $1.2 \times I_{c}$ is determined not only by the $I_{c}$ uniformity, but also by the uniformity of the normal zone dynamics.

Fig.4a shows that at $320 \mathrm{~A}\left(120 \%\right.$ of $\left.I_{c}\right)$ the initial dissipation, $V_{i n}, \approx 10^{-3} \mathrm{~V} / \mathrm{cm}$; it takes over $300 \mathrm{~ms}$ for the voltage to develop to the current-limiting level. We interpret $V_{i n}$, the 
voltage observed immediately upon application of the current step as dissipation of the instantly-created NZ, which is confirmed by the coincidence of the DCV(I) and $V_{\text {in }}(I)$ curves, shown in Fig.4b.In the following discussion we present a model which explains the wire response in the low-overcurrent regime and the role of the RF field. We make the simplifying assumptionthat a certain number of NZ's is instantly created after the application of the overcurrent and the following increase of the dissipation is explained by the growth and merging of NZ's.In the model we used experimentallydetermined NZPV, as shown in Fig.5.

In order to produce a noticeable current-limiting effect the normal zones require a characteristicresponse timein order to merge into continuous regions of the normal conductor. This response time, $t_{r}$, can be expressed as $t_{r}=d_{n} / v_{n}$, where $d_{n}$ is the average distance between the normal zones and $v_{n}$ is the NZPV. Our measurements of the propagation velocity,Fig.5b, confirm the observations of prior studies, which had demonstrated very slow NZ propagation in $2 \mathrm{G}$ wires[17, 22-24]. Low NZPV coupled with sparse normal zones lead to a very sluggish response of the wire to the overcurrent< $1.2 \times I_{c}$, Fig.6a. After the current application, only two sections, \#1 and \#4, develop noticeable dissipation and, according to Fig.5b, at $320 \mathrm{~A}$ it would take $\approx 200 \mathrm{~ms}$ for the $\mathrm{NZ}$ to propagate across the a $1 \mathrm{~cm}$ tape.

We use the following semi-empirical function to describe the dependence of $d_{n}$ on the current: $d_{n}=d_{\min } / e R F c\left(\left(I-I_{c l}\right) / \Delta I\right)$. Here, $d_{\min }$ is the minimum distance between stable normal zones, $I_{c l}$ is the fitting parameter proportional to the critical current density and $\Delta I_{c l}$ is the standard deviation of the $I_{c l}$ distribution along the tape. Here, $I_{c l}$ is a parameter (not necessarily equal to the experimental $I_{c}$ ) describing the statistical distribution of the 
normal zone density. The resulting dependence of the response time on the transport current is as follows:

$$
t_{r}=\frac{d_{\min }}{\operatorname{erfc}\left(\frac{I-I_{c 1}}{\Delta I_{c 1}}\right) \cdot v_{n}}
$$

TFA-MOD thick-film conductors, such as produced by AMSC, comprise large, 5-20 $\mu \mathrm{m}$, YBCO grains[25]. Magnetization studies have shown that the critical current inside a grain is $2-3$ times higher than the inter-grain current $[26,27]$. It is reasonable to assume that the minimum distance between the NZ's is equal to the grain size, i.e. $d_{m i n} \approx 5$ $\mu$ m.Eq.1 predicts that at $I<<I_{c l}$ there are very few normal zones and the response time is infinite. In the limit of very high over-currents, $I>>I_{c l}$, the speed of the conductor response is limited by the YBCO layer granularity, i.e. $t_{r}=d_{\min } / v_{n} \approx 50 \mu$ s.

The best fit of the response time, $t_{r}$, of the $2 \mathrm{G}$ wire in passive RF-assisted regime is shown in Fig.9. In the fitting procedure we used the experimentally-measured normal zone propagation velocity, Fig.5b, and varied $I_{c l}$ and $\Delta I_{c l}$. For the both sets of data the $\Delta I_{c l}$ value is quite large, $\approx 100 \mathrm{~A}$. The $I_{c l}$ value is $290 \mathrm{~A}$ for the passive mode and 275 A for the RF-assisted mode.Here we comment on the important difference between the $I_{c}$ and $I_{c l}$ parameters. The $I_{c}$ value is derived from the $I(V)$ curve using the "magnet builder" criterionof electric field strength on the order of $1 \mu \mathrm{V} / \mathrm{cm}$. In our case we are concerned with a dissipation level $>0.1 \mathrm{~V} / \mathrm{cm}$, which is $10^{5}$ times higher than the $I_{c}$ criterion. The $I_{c l}$ value and uniformity capture the dissipation dynamics in the current-limiting regime at much higher levels than $I_{c}$. In the current-limiting regime only rapidly expanding normal zones are contributing to the overall voltage. Apparently, not all the NZ's that are formed 
at $I_{c}$ are capable of fast expansion, which explains the high non-uniformity of the $I_{c l}$ distribution.

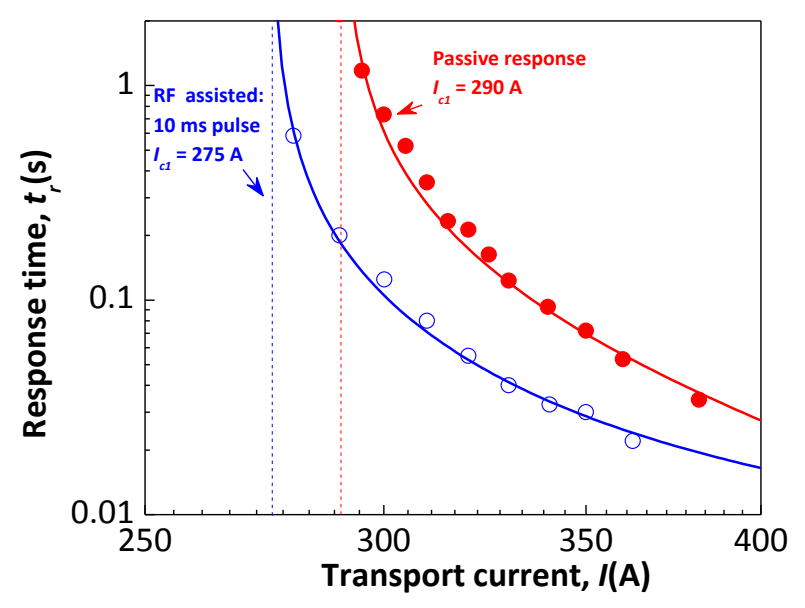

Fig.9. The response time of the $16 \mathrm{~cm}$ wire coupon as a function of the transport current in the passive mode (closed symbols) and assisted by a $10 \mathrm{msRF}$ pulse (open symbols). The solid lines are approximations by Eq. 1 . The vertical lines indicate $I_{c I}$ values determined from the best-fit approximations using Eq.1.

The approximations show that the faster response of the RF-assisted wire can be explained as an effectivereduction of the $I_{c l}$ by the RF field. The penetration of the RF field creates an inductive current which is superimposed on the transport current. This superposition increases the local current density above that of the local $J_{c}$ value, creating additional normal zones. If the RF field is applied long enough so that the normal zones produced by the RFsignal are stable and growing, the process will continue after the RF field is turned off.This model also explains the improved quench uniformity in the RFassisted regime, as shown in Fig.4b. Inductive currents are induced uniformly throughout the tape due to the strong coupling of the RF field with the superconductor, thus minimizing the effect of local variations of $J_{c}$ on the quench dynamics. 
The operation of the proposed device is different from the "magnetic-field triggered" FCL developed by Elschneret al. [28, 29]. The magnetic-field induced transition is realizable for melt-textured BiSCCO owing to low irreversibility field of the material at $77 \mathrm{~K}$. Thus, the application of moderate magnetic fields, $\approx 100 \mathrm{mT}$, can drive the melttextured BiSSCO rod into the normal state. This approach would be impractical for epitaxial YBCO with an irreversibility field larger than 5 T.The proposed device can be described as a high-frequency transformer with an efficient transfer of ac current from a normal coil into the superconducting element. Thus,the operation of the device is described as a current-induced, rather than field-induced, quench.

The improved uniformity of the RF-assisted quench allows sustaining higher voltages. $2 \mathrm{G}$ wires have complex structure, which can be irreversibly damaged by temperatureinduced stress. Measurements of the conductor behavior under unprotected quench show that $5 \%$ of $I_{c}$ is lost once the wire temperature reaches $490 \pm 50 \mathrm{~K}[30]$. This is explained by the observation that the thermal stress exceedsthe strain limit of the YBCO layer, $\approx$ $0.5 \%$. In the case of a laminated wire, the partial melting of the solder at $179^{\circ} \mathrm{C}$ is an additional factor contributing to the conductor failure. In the passive regime, the dissipation is concentrated in a few normal zones, resulting in a catastrophic overheating of the superconductor in those areas at $0.5 \mathrm{~V} / \mathrm{cm}$, Fig.7b. In contrast, in the RF-assisted mode the dissipation is distributed uniformly. This is why the tape can sustain repeated quenches without detectable degradation, Fig.7a.Thus, the RFcoil can also be described as a quench-protection device. It is well known that an unprotected quench of a superconducting magnet can result in localized damage to the superconducting wire [31]. A popular method of quench protection involves heaters embedded in the magnet 
winding [32]. The heaters, which are activated during the quench, distribute the dissipation over a large area, thus preventing localized overheating of the winding. The RFcoil is essentially performing the same function of protecting the FCL from localized damage during the quench.

Scale up of the technology to the distribution and transmission level voltages requires a change to the RFcoil geometry, since in an actual device the RFcoil would have to excite 10 's of meters of the conductor.In this work we have tested one possible solution, which replaced the racetrack coil with a single loop of multi-strand Litz wire, as shown in Fig.2. This geometry provides the same level of coupling as the racetrack coil and can be scaled up to hundreds of meters. Our tests of the prototype FCL device, Fig.8, show that a substantial improvement in response time can be attained on the several meter scale.

\section{Conclusion}

In conclusion, we evaluated the response of stainless-steel stabilized $2 \mathrm{G}$ wire to high-frequency inductive currents. We show that multiple normal zones can be created by short, $10 \mathrm{~ms}$, low-power bursts of high-frequency magnetic field.The presence of a high density of normal zones explains the faster and substantially more uniform response of the wire to the overcurrent, when application of the overcurrent is assisted by the RFfield. We tested the concept on a small scale FCL prototype, which demonstrated a factor of up to 60 faster current limiting compared with a traditional passive regime.Further work will focus on scaling-up of the design to higher currents and voltages and testing the device at $60 \mathrm{~Hz}$ AC. 


\section{Acknowledgements}

This manuscript has been authored by Brookhaven Science Associates, LLC under Contract No. DE-SC00112704with the U. S. Department of Energy.The work at Brookhaven National Laboratory was supported by Technology Maturation Grant TM 14-010. The authors wish to thank MartinRupich, Alex Malozemoff and Ivo Dimitrov valuable comments and corrections.

\section{References}

1. "2015 STATE OF THE ELECTRIC UTILITY." Survey by Utility Drive, Online: http://www.utilitydive.com/news/what-utility-execs-think-the-futureholds/356710/, 2015: p. 22.

2. "Utility of the Future Pulse Survey 2014." DNV GL Survey, Online: http://www.dnvgl.com/energy/download/U-of-F-Pulse-Survey-2014.aspx, 2014.

3. Yucheng, Z. and R.A. Dougal. State of the art of Fault Current Limiters and their applications in smart grid. in Power and Energy Society General Meeting, 2012 IEEE. 2012.

4. $\quad$ " Fault Current Limiters impact on protections." CIGRE Brochure 339, 2008.

5. Fault Current Limiters in Electrical Medium and High Voltage Systems. CIGRE Working Group 10 of Study Committee A3 (High Voltage Equipment)2003.

6. Elschner, S., J. Bock, and S. Gauss, Development of HTSC-rings for inductive fault current limiters. Advances in Superconductivity VII. Proceedings of the 7th International Symposium on Superconductivity, ed. K. Yamafuji and T. Morishita1995. 1211-14 vol.2. 
7. Fleshler, S., K. DeMoranville, J. Gannon, Jr., X. Li, Jr., E. Podtburg, M.W. Rupich, S. Sathyamurthy, C.L.H. Thieme, D. Tucker, and L. Whitman, "Development Status of AMSC Amperium reg Wire." Journal of Physics: Conference Series, 2014. 507(2): p. 022005 (4 pp.)-022005 (4 pp.).

8. Malozemoff, A.P., S. Fleshler, M. Rupich, C. Thieme, X. Li, W. Zhang, A. Otto, J. Maguire, D. Folts, J. Yuan, H.P. Kraemer, W. Schmidt, M. Wohlfart, and H.W. Neumueller, "Progress in high temperature superconductor coated conductors and their applications." Superconductor Science \& Technology, 2008. 21(3): p. 034005 .

9. Selvamanickam, V., Y. Chen, X. Xiong, Y.Y. Xie, J.L. Reeves, X. Zhang, Y. Qiao, K.R. Lenseth, R.M. Schmidt, A. Rar, D.W. Hazelton, and K. Tekletsadik, "Recent progress in second-generation HTS conductor scale-up at SuperPower." IEEE Transactions on Applied Superconductivity, 2007. 17(2): p. 3231-3234.

10. Xie, Y.Y., K. Tekletsadik, D. Hazelton, and V. Selvamanickam, "Second generation high-temperature superconducting wires for fault current limiter applications." Ieee Transactions on Applied Superconductivity, 2007. 17(2): p. 1981-1985.

11. Neumueller, H.W., W. Schmidt, H.P. Kraemer, A. Otto, J. Maguire, J. Yuan, D. Folts, W. Romanosky, B. Gamble, D. Madura, A.P. Malozemoff, N. Lallouet, S.P. Ashworth, J.O. Willis, and S. Ahmed, "Development of Resistive Fault Current Limiters Based on YBCO Coated Conductors." Ieee Transactions on Applied Superconductivity, 2009. 19(3): p. 1950-1955. 
12. "AMSC, Siemens, Nexans; "Industry Leaders Successfully Demonstrate Transmission Voltage Superconductor Fault Current Limiter - American Superconductor, Nexans and Siemens Successfully Test High Voltage System”, October, $2011 \quad$ [Online], available: http://www.nexans.com/Corporate/2011/Nexans_FCL_Testing_GB.pdf.".

13. Bourne, J., M. Schupbach, J. Carr, H.A. Mantooth, and J. Balda. Initial development of a solid-state fault current limiter for naval power systems protection. in Electric Ship Technologies Symposium, 2009. ESTS 2009. IEEE. 2009.

14. The Future of the Electric Grid, 2011, Massachusetts Institute of Technology, Online at http://web.mit.edu/mitei/research/studies/the-electric-grid-2011.shtml.

15. Solovyov, V.F. and Q. Li, "Fast high-temperature superconductor switch for high current applications." Applied Physics Letters, 2013. 103(3): p. 032603-3.

16. Wang, X., U.P. Trociewitz, and J. Schwartz, "Near-adiabatic quench experiments on short YBa2Cu3O7- $\delta$ coated conductors." Journal of Applied Physics, 2007. 101(5): p. - .

17. Ishiyama, A., M. Yanai, T. Morisaki, H. Ueda, Y. Shiohara, T. Izumi, Y. Iijima, and T. Saitoh, "Normal transition and propagation Characteristics of YBCO tape." Applied Superconductivity, IEEE Transactions on, 2005. 15(2): p. 16591662.

18. Manfreda, G., F. Bellina, and G. Volpini, "Critical current statistical distribution and voltage-current characteristics in superconducting wires." Superconductor Science and Technology, 2014. 27(12): p. 125005. 
19. Jones, R.G., E.H. Rhoderick, and A.C. Rose-Innes, "Non-linearity in the voltagecurrent characteristic of a type-2 superconductor." Physics Letters A, 1967. 24(6): p. 318-319.

20. Gannon, J.J., Jr., A.P. Malozemoff, R.C. Diehl, P. Antaya, and A. Mori, "Effect of Length Scale on Critical Current Measurement in High Temperature Superconductor Wires." Ieee Transactions on Applied Superconductivity, 2013. 23(3).

21. Wen, J., B. Lin, J. Sheng, J. Xu, Z. Jin, Z. Hong, D. Wang, H. Zhou, X. Shen, and C. Shen, "Maximum permissible voltage of YBCO coated conductors." Physica CSuperconductivity and Its Applications, 2014. 501: p. 14-18.

22. George, A.L. and N.B. Paul, "The normal zone in $\mathrm{YBa} 2 \mathrm{Cu} 3 \mathrm{O} 6+x$-coated conductors." Superconductor Science and Technology, 2007. 20(12): p. 1101.

23. Trillaud, F., H. Palanki, U.P. Trociewitz, S.H. Thompson, H.W. Weijers, and J. Schwartz, "Normal zone propagation experiments on HTS composite conductors." Cryogenics, 2003. 43(3-5): p. 271-279.

24. Bellis, R.H. and Y. Iwasa, "Quench propagation in high Tc superconductors." Cryogenics, 1994. 34(2): p. 129-144.

25. Solovyov, V., I. Dimitrov, K., and Q. Li, "Growth of thick $\mathrm{YBa}_{2} \mathrm{Cu}_{3} \mathrm{O}_{7}$ layers via a barium fluoride process." Superconductor Science and Technology, 2013. 26(1): p. 013001.

26. Palau, A., T. Puig, X. Obradors, R. Feenstra, A.A. Gapud, E.D. Specht, D.M. Feldmann, and T.G. Holesinger, "Grain and grain-boundary critical currents in 
coated conductors with noncorrelating YBa2Cu3O7 and substrate grainboundary networks." Applied Physics Letters, 2006. 88(13): p. - .

27. Palau, A., T. Puig, X. Obradors, R. Feenstra, and A.A. Gapud, "Correlation between grain and grain-boundary critical current densities in ex situ coated conductors with variable YBa2Cu3O7-delta layer thickness." Applied Physics Letters, 2006. 88(12): p. -.

28. Elschner, S., F. Breuer, H. Walter, and J. Bock, "Magnetic Field Assisted Quench Propagation as a New Concept for Resistive Current Limiting Devices." 7th European Conference on Applied Superconductivity (Eucas'05), 2006. 43: p. $917-$ 920.

29. Elschner, S., F. Breuer, H. Walter, M. Stemmle, and J. Bock, "HTS components for high voltage resistive current limiters based on a magnetic field triggered concept." Ieee Transactions on Applied Superconductivity, 2007. 17(2): p. 17721775.

30. Wang, $\mathrm{X}$. and et al., "Critical current degradation of short YBa $2 \mathrm{Cu} 3 \mathrm{O} 7-\delta$ coated conductor due to an unprotected quench." Superconductor Science and Technology, 2011. 24(3): p. 035006.

31. Wilson, M.N., Superconducting magnets. Monographs on cryogenics [2]1983, Oxford Oxfordshire

New York: Clarendon Press; Oxford University Press. xv, 335 p.

32. Schultz, J.H., "Protection of superconducting magnets." Applied Superconductivity, IEEE Transactions on, 2002. 12(1): p. 1390-1395. 